\title{
Hypoxic-Ischemic Injury of Basal \\ Ganglia Associated with the COVID-19 Infection: Case Report
}

\author{
Cyprian Popescu
}

Department of Neurology, Victor Pauchet Clinic, Amiens, France

\section{Keywords}

SARS-CoV-2 COVID-19 · Hypoxic-ischemic injury of basal ganglia · Diffusion-weighted image

\begin{abstract}
There is emerging evidence of a broad spectrum of neurological manifestations in COVID-19 patients. We report a case of a patient diagnosed with COVID-19 who presented bilateral lesions of the basal ganglia related to a severe acute respiratory distress syndrome. This observation allows expanding the neurological spectrum of COVID-19 particularly in patients with a more severe clinical course.
\end{abstract}

\section{Case Report}

A 56-year-old French woman without previous neurological history and a past medical history of hypothyroidism and hyperlipidemia presented with a 7-day history of febrile state and dyspnea. She was diagnosed with pneumonia linked to COVID-19. Because her respiratory condition deteriorated, endotracheal intubation and mechanical ventilation were required for 18 days. A treatment with azithromycin, cefotaxime, and hydroxychloroquine was initiated. Due to the worsening of the situation toward a severe acute respiratory distress syndrome, profound sedation and a treatment with lopinavir/ritonavir were implemented. She developed a septic shock and an acute pulmonary edema during the ventilatory withdrawal. Blood investigation showed an abnormality in liver function indexes. After extubating, the patient progressively recovered but not completely from respiratory symptoms with persistent mild dyspnea and desaturation with exercise. Routine blood test including white blood cell and blood platelet 
Popescu: Hypoxic-Ischemic Injury of Basal Ganglia Associated with the COVID-19 Infection

Fig. 1. Diffusion-weighted imaging reveals signal intensity abnormalities in the bilateral putamen.

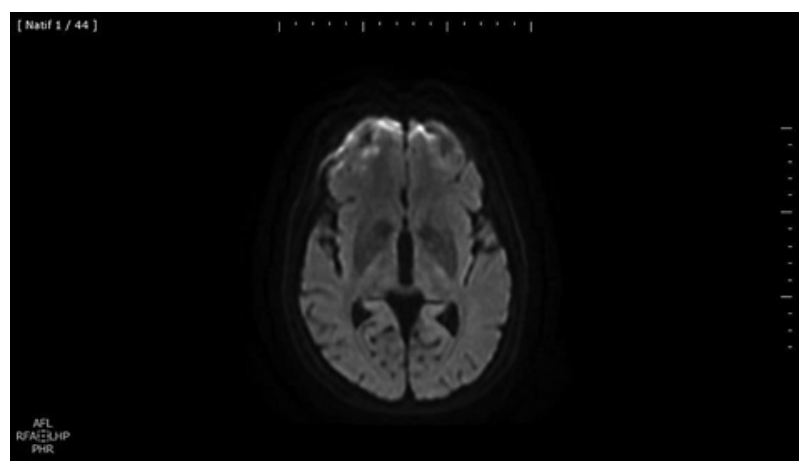

count was normal. Renal (blood urea nitrogen and serum creatinine) and hepatic function indexes (alanine transaminase and aspartate transaminase) were normalized. Electrolytes were serum $\mathrm{K} \mathrm{(+)} 4.1 \mathrm{mmol} / \mathrm{L}, \mathrm{Na} \mathrm{(+)} 137 \mathrm{mmol} / \mathrm{L}$, and CRP level $9 \mathrm{mg}$. Coagulation function including prothrombin time, international normalized ratio, and thrombin time was normal. Blood gas analysis under $2 \mathrm{~L} / \mathrm{min}$ oxygen showed $\mathrm{pCO}_{2} 46.7 \mathrm{~mm} \mathrm{Hg}, \mathrm{pO}_{2} 52.6 \mathrm{~mm} \mathrm{Hg}$, pH value 7.396, saturation $85 \%$, and lactate $2.6 \mathrm{mmol} / \mathrm{L}$. ECG was normal without repolarization changes. Transthoracic echocardiogram shows a normal ejection fraction; atrial and ventricular size and morphology were also normal. Chest CT shows bilateral and peripheral predominant groundglass opacities. On examination, 4 months after the onset of disease, the patient had complaints of headache and concentration difficulties. There was no impairment of cognitive functions, including executive functions. Besides concentration troubles, she had no more amnesic and aphasic features and no more dysphonia. Verbal fluency was mildly reduced, and test TMT-A and B, the five-word test (FWT), and the clock-drawing test were normal. There were no parkinsonian syndrome, micrographia, rigidity, bradykinesia, or tremor. Her gait and arm swing was normal. Four months after the insult, the patient underwent EMG which was judged to be normal and an MRI. The diffusion-weighted image revealed residual decreased signal intensity of the bilateral putamen (Fig. 1). Because of the involvement of striatal structures, a DaT-SPECT was performed which did not show a decrease in presynaptic dopamine uptake.

\section{Discussion}

Previous reports have addressed aspects of a wide range of neurologic complications of COVID-19 including impairment of underlying neurologic conditions. It has been suggested as possible mechanisms of brain structure lesions a direct viral nervous system invasion or an aggressive inflammatory response induced by the cytokine release syndrome [1]. There is a growing body of published evidence that a hypercoagulable state seems to be associated with disease severity [1]. We report a case of a patient with bilateral lesions of the basal ganglia in a severe infectious and hypoxic-ischemic context. Therefore, the first hypothesis is that of an ischemic/hypoxic encephalopathy linked to the COVID-19 infection. The pathophysiologic basis of basal ganglia involvement includes hypoxic ischemic or hepatic encephalopathy inhibiting the respiratory chain metabolic and infectious causes. Basal ganglia have high metabolic requirement, and therefore hypoxic-ischemic injury may trigger intracellular cytotoxic edema and restricted diffusion. Encephalopathy associated with SARS-CoV-2 infection and bilateral thalamic involvement was already described in a 33-month-old boy with multisystem inflammatory syndrome in children [2]. Bilateral globus pallidus lesions attributed to hypoxic-ischemic injury have already been described in a patient with SARS-CoV-2 [3]. The imaging features in

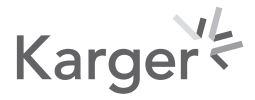


our case are also interpreted as global hypoxia and less likely as leptomeningeal invasion because of the symmetric pattern of basal ganglia involvement. The septic shock and therefore hypovolemia and metabolic acidosis is the most plausible cause of ischemic/hypoxic basal ganglia injury. MR images obtained 4 months after the infection depict symmetric hypointensity of the bilateral putamen and high signal intensity foci in the subcortical white matter consistent with small vessel involvement. Restricted diffusion of basal ganglia by diffusion-weighted imaging could be secondary to metal intoxication such as manganese, but the anamnesis has already excluded it. In our case, an abnormality in liver function was noted, but no hyperammonemic encephalopathy features were signaled. Discordance between restricted diffusion in the bilateral lateral thalamic nuclei and T2/fluid-attenuated inversion recovery changes was already described in a COVID-19 child [2]. Diffusion abnormalities involving basal ganglia could be the result of the disruption of oxygen delivery to the brain with the preservation of the blood flow [4]. In contrast, the associated punctiform lesions as potentially ischemic lesions might stand for small-vessel vasculitis. In our case, DaT-SPECT shows a normal putaminal dopaminergic function, whereas a bilateral decrease in presynaptic dopamine uptake in the striatum was described in a patient with severe infection [5]. The absence of delayed neurological sequelae does not necessarily exclude an early extrapyramidal involvement with characteristic imaging findings such as reduced diffusivity in the acute phase. Acute infection inducing microglial activation and infiltration of cytotoxic T lymphocyte [1] might be consistent with toxic stress but not sufficient to induce neurodegeneration and neuronal death if there are strong neuroprotective responses. The hypoxic-ischemic hypothesis is supported by the neuropathologic data with mild hypoxemic cerebral changes [6]. This observation allows expanding the neurological spectrum of COVID-19 particularly in patients with a more severe clinical course.

\section{Statement of Ethics}

The subject gave her written informed consent to publish her case including publication of images. The author confirms that the approval of an institutional review board was not required for this work. Furthermore, the author confirms to have accepted the journal's position on issues involved in ethical publication and affirms that this work is consistent with those guidelines.

\section{Conflict of Interest Statement}

The author declares that the research was conducted in the absence of any commercial or financial relationships that could represent a potential conflict of interest.

\section{Funding Sources}

No funding was received.

\section{Data Availability Statement}

The datasets used and/or analyzed during the current study are available from the corresponding author, upon reasonable request. The data are not generally accessible because they contain information that could compromise the privacy of the research participant.

\section{Karger's}




\section{References}

1 Mao L, Jin H, Wang M, Hu Y, Chen S, He Q, et al. Neurologic manifestations of hospitalized patients with Coronavirus disease 2019 in Wuhan, China. JAMA Neurol. 2020 Jun 1;77(6):683-90.

2 Abel D, Shen MY, Abid Z, Hennigan C, Boneparth A, Miller EH, et al. Encephalopathy and bilateral thalamic lesions in a child with MIS-C associated with COVID-19. Neurology. 2020 Oct 20;95(16):745-8.

3 Kulick-Soper CV, McKee JL, Wolf RL, Mohan S, Stein JM, Masur JH, et al. Pearls \& Oy-sters: bilateral globus pallidus lesions in a patient with COVID-19. Neurology. 2020 Sep 8;95(10):454-7.

4 Singhal AB, Topcuoglu MA, Koroshetz WJ. Diffusion MRI in three types of anoxic encephalopathy. J Neurol Sci. 2002;196:37-40.

5 Méndez-Guerrero A, Laespada-García MI, Gómez-Grande A, Ruiz-Ortiz M, Blanco-Palmero VA, Azcarate-Diaz FJ, et al. Acute hypokinetic-rigid syndrome following SARS-CoV-2 infection. Neurology. 2020 Oct 13;95(15): e2109-18.

6 Menter T, Haslbauer JD, Nienhold R, Savic S, Hopfer H, Deigendesch N, et al. Postmortem examination of COVID-19 patients reveals diffuse alveolar damage with severe capillary congestion and variegated findings in lungs and other organs suggesting vascular dysfunction. Histopathology. 2020 Aug;77(2):198-209. 